\title{
Band structure of cavity-type hypersonic phononic crystals fabricated by femtosecond laser-induced two-photon polymerization
}

A. M. Rakhymzhanov, A. Gueddida, E. Alonso-Redondo, Z. N. Utegulov, D. Perevoznik, K. Kurselis, B. N. Chichkov, E. H. El Boudouti, B. Djafari-Rouhani, and G. Fytas

Citation: Appl. Phys. Lett. 108, 201901 (2016); doi: 10.1063/1.4949013

View online: https://doi.org/10.1063/1.4949013

View Table of Contents: http://aip.scitation.org/toc/apl/108/20

Published by the American Institute of Physics

\section{Articles you may be interested in}

Direction-dependent elastic properties and phononic behavior of $\mathrm{PMMA}_{\mathrm{BaTiO}}$ nanocomposite thin films

The Journal of Chemical Physics 146, 203325 (2017); 10.1063/1.4978675

Hypersonic phonon propagation in one-dimensional surface phononic crystal

Applied Physics Letters 104, 123108 (2014); 10.1063/1.4870045

Low-frequency band gap and defect state characteristics in a multi-stub phononic crystal plate with slit structure Journal of Applied Physics 121, 015106 (2017); 10.1063/1.4973577

Finite element analysis of true and pseudo surface acoustic waves in one-dimensional phononic crystals Journal of Applied Physics 119, 025308 (2016); 10.1063/1.4939825

Modeling and experimental verification of an ultra-wide bandgap in 3D phononic crystal Applied Physics Letters 109, 221907 (2016); 10.1063/1.4971290

Thermal phonon transport in silicon nanowires and two-dimensional phononic crystal nanostructures Applied Physics Letters 106, 143102 (2015); 10.1063/1.4917036

\section{Conference Proceedings}




\title{
Band structure of cavity-type hypersonic phononic crystals fabricated by femtosecond laser-induced two-photon polymerization
}

\author{
A. M. Rakhymzhanov, ${ }^{1,2}$ A. Gueddida, ${ }^{3,4}$ E. Alonso-Redondo, ${ }^{5}$ Z. N. Utegulov, ${ }^{1,2, a)}$ \\ D. Perevoznik, ${ }^{6}$ K. Kurselis, ${ }^{6}$ B. N. Chichkov, ${ }^{6,7}$ E. H. El Boudouti, ${ }^{4}$ B. Djafari-Rouhani, ${ }^{3}$ \\ and G. Fytas ${ }^{5,8, a)}$ \\ ${ }^{1}$ Department of Physics, School of Science and Technology, Nazarbayev University, Astana 010000, \\ Kazakhstan \\ ${ }^{2}$ Optics Laboratory, National Laboratory Astana, Nazarbayev University, Astana 10000, Kazakhstan \\ ${ }^{3}$ Institut d' Electronique, Microélectronique et Nanotechnologie, 59650 Villeneuve d' Ascq, France \\ ${ }^{4}$ LPMR, Département de Physique, Faculté des Sciences, Université Mohamed I, 60000 Oujda, Morocco \\ ${ }^{5}$ Max Planck Institute of Polymer Research, Ackermannweg 10, 55128 Mainz, Germany \\ ${ }^{6}$ Laser Zentrum Hannover e.V., 30419 Hannover, Germany \\ ${ }^{7}$ Institute of Laser and Information Technologies RAS, Moscow, 142092 Troitsk, Russia \\ ${ }^{8}$ Department of Materials Science, University of Crete and FORTH, 71110 Heraklion, Greece
}

(Received 5 February 2016; accepted 28 April 2016; published online 16 May 2016)

The phononic band diagram of a periodic square structure fabricated by femtosecond laser pulseinduced two photon polymerization is recorded by Brillouin light scattering (BLS) at hypersonic $(\mathrm{GHz})$ frequencies and computed by finite element method. The theoretical calculations along the two main symmetry directions quantitatively capture the band diagrams of the air- and liquid-filled structure and moreover represent the BLS intensities. The theory helps identify the observed modes, reveals the origin of the observed bandgaps at the Brillouin zone boundaries, and unravels direction dependent effective medium behavior. Published by AIP Publishing.

[http://dx.doi.org/10.1063/1.4949013]

Phononic crystals (PnC), the acoustic analogues of photonic crystals, are periodic structures made of materials with different acoustic impedances. The main consequence of the structure periodicity is the formation of band gaps in the phonon dispersion at certain wavelengths that commensurate with the lattice constant. PnC with periodicity in the submicrometer range can block hypersonic phonons with potential applications in compact wireless and sensing devices. ${ }^{1,2}$ Control over the $\mathrm{GHz}$ phonons in polymers and semiconductors could lead to the design of new highly efficient hybrid photonic-phononic signal processing technologies. ${ }^{3}$ Devices in the optoelectronics industry such as resonators, cavities, and waveguides can be realized in a phononic crystal by removal or distortion of the inclusions. ${ }^{1,4}$

Since the first realization of a hypersonic phononic band gap, ${ }^{5}$ the design, fabrication, and characterization of $1 \mathrm{D}, 2 \mathrm{D}$, and 3D hypersonic phononic crystals $(\mathrm{hPnC})$ have been the subject of intensive research. ${ }^{6-14}$ However, the fabrication of such periodic crystals is still a technological challenge, since the control over the periodicity for various 3D architectures on the sub micrometer scale is necessary. Electron and focused ion-beam lithographic techniques are powerful in producing 2D GHz phononic structures in opaque media. ${ }^{15,16}$ Selfassembly by vertical lifting deposition ${ }^{5,7}$ and holographic interference lithography ${ }^{6,17,18}$ have enabled the fabrication of $2 \mathrm{D}$ $\mathrm{hPnC}$ in transparent polymeric media with limited 3D architectures. To realize true multiple 3D nano- and micro-structured structures in photosensitive polymeric media, direct laser writing technology based on the multi-photon absorption processes

\footnotetext{
${ }^{\text {a) }}$ Authors to whom correspondence should be addressed. Electronic addresses: zhutegulov@nu.edu.kz and fytas@mpip-mainz.mpg.de
}

such as two-photon polymerization (2PP) is a very attractive option. ${ }^{19-21}$ Because of the threshold behavior and nonlinear nature of the $2 \mathrm{PP}$ process, small spatial features with optical resolution beyond the diffraction limit can be achieved. By scanning the focused laser spot inside a photo-resist, various predefined 3D topographic shapes can be formed. So far, 2PP technique has been applied for the fabrication of $2 \mathrm{D}$ and $3 \mathrm{D}$ photonic crystals, ${ }^{22-27}$ but we are not aware of its utilization in phononics. In this work, we use the femtosecond laser 2PP to fabricate square lattice $\mathrm{hPnCs}$ based on the zirconium propoxide (ZPO)-based hybrid sol-gel photosensitive material. ${ }^{26}$

There are few reports on cavity-type $\mathrm{hPnCs}$ with cylindrical nanopores, e.g., with a single crystalline epoxy/air sample, ${ }^{18}$ anodic porous alumina containing hexagonal arrays of aligned nanopores oriented normal to the film surface, ${ }^{27,28}$ and square arrays of holes of Si-membranes. ${ }^{29}$ Using Brillouin light scattering spectroscopy (BLS) in transmission geometry (see Fig. 2(d)), the phonon dispersion relation has been recorded for a square lattice $\mathrm{hPnC}$ with filled spherical nanopores ${ }^{6}$ and hexagonal lattice with both air- and filled spherical nanopores. ${ }^{30}$ In the former case, there was no direct comparison with the theoretical band diagram, while in the latter this comparison was restricted on the observed phononic branches. For the semi- or non-transparent $\mathrm{hPnCs},{ }^{28,29} \mathrm{BLS}$ was employed in backscattering geometry where the ripple mechanism dominated the scattering from the surface. For a fundamental understanding, however, the theoretical representation of the full BLS spectrum, frequencies, and intensities is necessary. Here, we report on the experimental band diagram of both air and filled square nanopores in a square lattice recorded by BLS and theoretical calculations of the dispersion and the BLS intensities. 
The band structure of the square lattice polymer-based $\mathrm{hPnC}$ is sensitive to the pore shape as illustrated in Figure 1 for air-filled holes, lattice parameter $a=740 \mathrm{~nm}$, filling fraction $f=0.46$, and elastic properties representing the $\mathrm{hPnC}$ of this study. This problem was studied theoretically for periodic fibre reinforced composite solid materials ${ }^{31}$ and solid square rods in air ${ }^{32}$ enabling tunability of the band gap by rotating the rods. The effect of the inclusion shapes or varying geometry in architectured materials is of current research interest. ${ }^{33}$ The phonon dispersion curves are calculated for a 2D crystal being infinite along the $z$ direction to avoid superposition with the numerous modes propagating in the substrate and represent the in-plane modes polarized in the $(\mathrm{x}, \mathrm{y})$ plane. The calculation is performed by using finite element method and the dispersion curves present the frequency as a function of the Bloch wave vector $\mathbf{k}=\left(k_{\mathrm{x}}, k_{\mathrm{y}}\right)$ along the high symmetry axes ( $\Gamma \mathrm{X}$ and $\Gamma \mathrm{M})$ of the Brillouin zone (BZ). The band structures for square lattice symmetry clearly depend on whether the inclusions are circular or rotatedsquare shapes (Figs. 1(b) and 1(c)). Absolute band gap can be opened in the rotated-square structure and even in the circular geometry, whereas the non-rotated square shape yields partial band gaps (along $\Gamma \mathrm{M}$ ).

To realize such periodic structures, we synthesized the photoresist ${ }^{26}$ and used it as a platform for its subsequent polymerization with lateral 2D periodicity. The focal spot of the writing pulse laser beam $(517 \mathrm{~nm}, 60 \mathrm{fs}, 76 \mathrm{MHz}, 70$ $\mathrm{mW})$ was accurately scanned $(0.2 \mathrm{~mm} / \mathrm{s}$ in a vertical and lateral plane of the transparent pre-baked $\mathrm{Zr}$-propoxide (ZPO) photoresist). ${ }^{34}$ Fabrication of a $1 \times 1 \mathrm{~mm}^{2}$ sample required about $8 \mathrm{~h}$. To remove the non-polymerized material, the sample was developed in 1-propanol. Top view scanning electron micrograph of the $2 \mathrm{D}-\mathrm{hPnC}$ by $2 \mathrm{PP}$ is shown in Figure 2(a) along with the schematic picture in Figure 2(c) yielding a filling fraction, $f=0.46$. The two main crystallographic directions $\Gamma \mathrm{M}(110)$ and $\Gamma \mathrm{X}(100)$ are indicated by the arrows.

The phonon band structure of the air-filled and infiltrated $\mathrm{hPnC}$ was characterized by BLS spectroscopy. It is based on the elasto-optic interaction of the incident light with thermally activated phonons that results in a Brillouin double shift $( \pm \omega)$ in the spectrum of homogeneous media. Nanostructured media display much richer spectrum (Fig. 3(a)) represented by a sum of Lorentzian lines and their peak position defines the phonon frequencies at a given wavenumber $q .{ }^{5,7,12,14}$ In the present $\mathrm{hPnC}$, phonons were probed with wave vector $\mathbf{q}=\mathbf{k}_{\mathbf{s}}-\mathbf{k}_{\mathbf{i}}$ (Fig. 2(d)) lying along
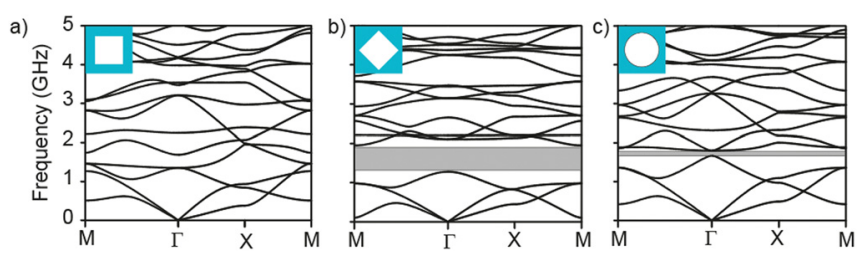

FIG. 1. Dispersion curves of square lattice polymer based phononic crystal with square (a), rotated-square (b), and circular (c) holes. The lattice parameter, filling fraction, and density are, respectively, $a=740 \mathrm{~nm}, f=0.46$, and $\rho=1190 \mathrm{~kg} / \mathrm{m}^{3}$, whereas the longitudinal and transverse sound velocities in the matrix assume $c_{\mathrm{L}}=2900 \mathrm{~m} / \mathrm{s}$ and $c_{\mathrm{T}}=1700 \mathrm{~m} / \mathrm{s}$. a)

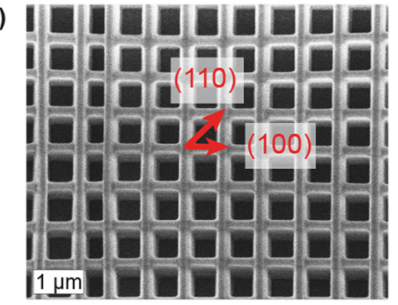

c)

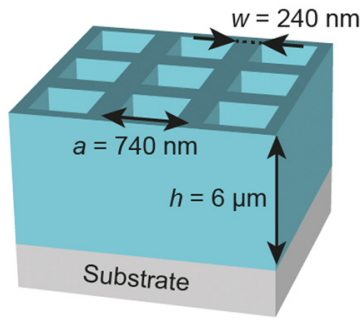

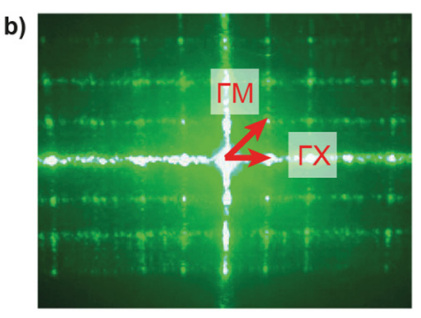

d) Incident light

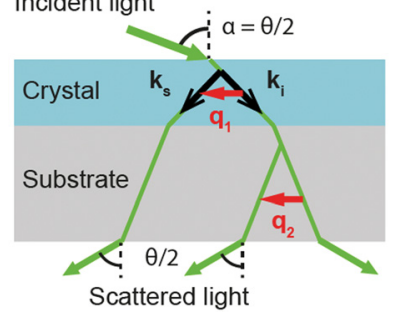

FIG. 2. Scanning electron micrograph (a) (top view) and schematic representation (c) of the structure with the two main crystallographic directions indicated by the red arrows in (a). (b) Photograph of the optical diffracted pattern with the two symmetry directions marked by the red arrows. (d) Lateral view of the Brillouin scattering geometry with $\mathbf{k}_{\mathrm{i}}, \mathbf{k}_{\mathrm{s}}$, and $\mathbf{q}_{\mathbf{1 , 2}}$ being the wave vectors of the incident and scattered light, and probed phonon $\left(\left|\mathbf{q}_{1}\right|=\left|\mathbf{q}_{2}\right|\right)$, respectively. The thickness of glass substrate in (c) and (d) is not to scale.

the $\Gamma \mathrm{X}$ and $\Gamma \mathrm{M}$ (Fig. 1(b)); $\mathbf{k}_{\mathbf{i}}\left(\mathbf{k}_{\mathbf{s}}\right)$ is the incident (scattered) light wave vector. The magnitude, $q=4 \pi / \lambda \sin (\theta / 2)$, is determined by the angle $\theta$ between $\mathbf{k}_{\mathbf{i}}$ and $\mathbf{k}_{\mathbf{s}}$ with $\lambda=532 \mathrm{~nm}$ being the wavelength of the laser beam. Note that for the transmission geometry of Figure 2(d) (with $\theta=2 \alpha$ ), there is a complete cancellation of the refractive index ( $n$ ) of the medium and hence $\mathbf{q}$ does not depend on $n .{ }^{35}$ Figure 2(b) displays the image of the diffracted pattern of the laser beam impinging on $\mathrm{hPnC}$ and the red arrows (in Fig. 2(b)) mark the direction ( $Г \mathrm{X}$ or $Г \mathrm{M})$ of the probing wave vector $\mathbf{q}$.

For the air-filled $\mathrm{hPnC}$, the dispersion relation was recorded only along $\Gamma \mathrm{M}$ due to very strong elastic (Rayleigh) scattering along $\Gamma \mathrm{X}$. Experimental BLS spectra at $q<q_{\Gamma \mathrm{M}} \sim 6$ $\times 10^{-3} \mathrm{~nm}^{-1}$, represented by Lorentzian lines, are shown in Figure 3(a). Since an independent access to the elastic properties of the unstructured material was not feasible, we rely on their determination searching for the optimal theoretical description of the full BLS spectra, i.e., dispersion, polarization, and relative peak intensities. As the spectra were recorded with $q$ 's extending beyond the first BZ, we show the unfolded dispersion relation into the second BZ in Figure 3(b) and Figure $\mathrm{S} 1{ }^{36}$ The branches are colored according to their polarization (longitudinal, L, vs. transverse, T) character. Based on the band sorting method,

$$
P_{X}=\frac{\iint_{u . c e l}\left|u_{X}\right|^{2} d S}{\iint_{\text {u.cel }}\left(\left|u_{X}\right|^{2}+\left|u_{Y}\right|^{2}\right) d S},
$$

where $\mathrm{X}$ is a direction parallel to the wave vector and $\mathrm{u}_{\mathrm{X}}$ represents the projection of the displacement field along this direction; [1,1] for $\Gamma \mathrm{M}$ and $[1,0]$ for $\Gamma X$. For $\mathrm{P}_{\mathrm{X}}$ close to $1(0)$, the corresponding mode has predominantly longitudinal (transverse) polarization; the value of $\mathrm{P}_{\mathrm{X}}$ is 

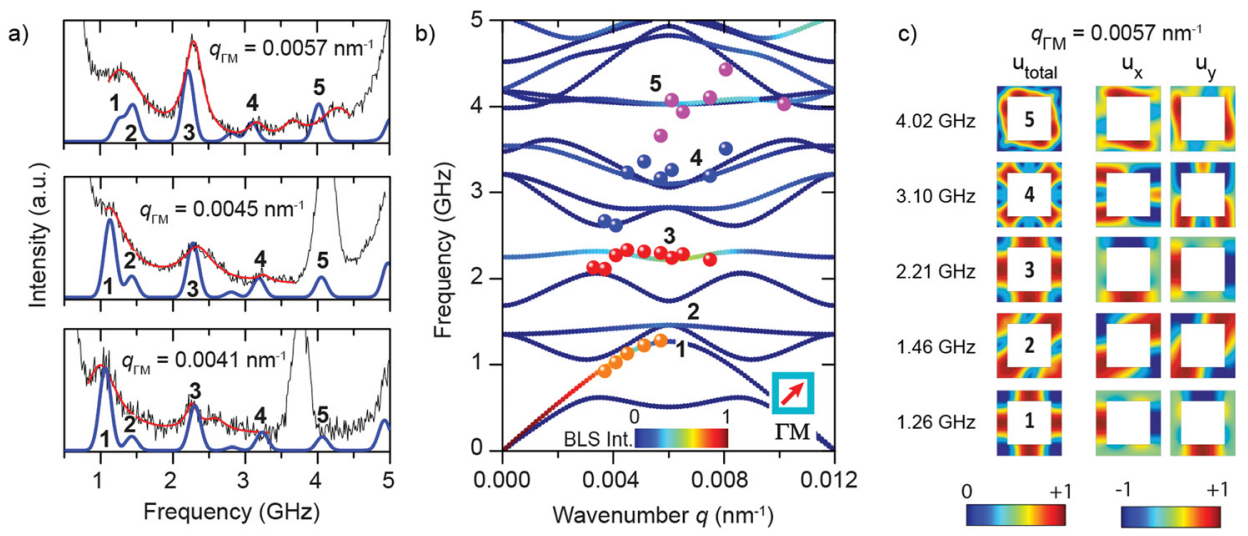

FIG. 3. (a) Experimental (black) and theoretical (blue) BLS spectra of the hPnC with air-filled square nanopores at three wave vectors, $q$ 's, along the $\Gamma M$ direction. The very strong peaks in the experimental spectra at the two lowest $q$ 's (around 3.8 and $4.1 \mathrm{GHz}$ ) belong to the longitudinal phonon in the glass substrate. The experimental spectra were represented by Lorentzian shapes (red lines). (b) Theoretical (lines) and experimental (dots) band diagrams along $\Gamma \mathrm{M}$ direction. The color scale denotes the strength of the BLS intensity. (c). Displacement fields for the modes at the wave number $q_{\Gamma M}=0.0057 \mathrm{~nm}^{-1}$ with increasing frequency. The color bars indicate the amplitude of the displacement.

highlighted by a color bar in Figure S1. The dispersion curves are given for two different choices of the acoustic velocities: $c_{\mathrm{L}}=2900 \mathrm{~m} / \mathrm{s}, c_{\mathrm{T}}=1400 \mathrm{~m} / \mathrm{s}$ in Figure S1(a), and $c_{\mathrm{L}}=2900 \mathrm{~m} / \mathrm{s}, c_{\mathrm{T}}=1700 \mathrm{~m} / \mathrm{s}$ in Figure S1(c). While both choices lead to a good agreement with the experimental phonon dispersion, the higher $c_{\mathrm{T}}$ appears more satisfactorily because it captures the longitudinal character of the observed modes. The value of $c_{\mathrm{L}}$ in the range of 2700-2900 $\mathrm{m} / \mathrm{s}$ does not influence the agreement between the theoretical and experimental dispersion curves.

The uniqueness of the velocity values can be verified by a more stringent criterion, which is the prediction of the BLS intensity. ${ }^{12,37}$ Since the samples are transparent, we use a simple expression of the Brillouin intensity based only on the elasto-optic (or photoelastic) mechanism of light-matter interaction, ${ }^{38}$

$$
I=\left|E_{j}^{s}\right|^{2} \propto\left|\frac{1}{\omega} \iint_{\text {unit.cell }} e^{i \vec{q} r} \sum_{i k l} P_{i j k l} S_{k l} E_{i}^{I} d S\right|^{2},
$$

where $E_{j}^{s}$ is the $j$ component of the scattered electric field, $E_{i}^{I}$ is the $i$ component of the electric field of the laser beam, $\mathrm{q}$ is the wave vector, $P_{i j k l}$ are the components of the photoelastic tensor, and $S_{k l}$ are the components of the strain tensor. For acoustic waves propagating along the $\Gamma \mathrm{M}$ direction and the VV scattering geometry of BLS, the above expression yields

$$
\begin{aligned}
\mathrm{I}_{\Gamma M}= & \left|\mathrm{E}^{\mathrm{s}}\right|^{2} \propto \mid \frac{1}{\omega} \iint_{\text {unit.cell }} \mathrm{e}^{\mathrm{i} \overrightarrow{\mathrm{q}} \overrightarrow{\mathrm{r}}}\left[\left(\mathrm{P}_{11}+\mathrm{P}_{12}\right) \times\left(\frac{\partial \mathrm{u}_{\mathrm{x}}}{\partial \mathrm{x}}+\frac{\partial \mathrm{u}_{\mathrm{y}}}{\partial \mathrm{y}}\right)\right. \\
& \left.-2 \times \mathrm{P}_{66} \times\left(\frac{\partial \mathrm{u}_{\mathrm{x}}}{\partial \mathrm{y}}+\frac{\partial \mathrm{u}_{\mathrm{y}}}{\partial \mathrm{x}}\right)\right]\left.\mathrm{E}^{\mathrm{I}} \mathrm{dS}\right|^{2} .
\end{aligned}
$$

The photoelastic coefficients involved in this expression are $\mathrm{P}_{11}+\mathrm{P}_{12}$ and $\mathrm{P}_{66}$ where in the case of isotropic materials, they are related by $\mathrm{P}_{11}-\mathrm{P}_{12}=2 \mathrm{P}_{66}$. To compare with the relative intensities in the BLS spectra, there is only the ratio $\mathrm{P}_{66} /\left(\mathrm{P}_{11}+\mathrm{P}_{12}\right)$, which is used as a free parameter. The best qualitative agreement was found when $\mathrm{P}_{66} /\left(\mathrm{P}_{11}+\mathrm{P}_{12}\right)$ is negative and relatively small (about $-0.2, \mathrm{P}_{11} /\left(\mathrm{P}_{11}+\mathrm{P}_{12}\right)=0.3$ and $\mathrm{P}_{12} /\left(\mathrm{P}_{11}+\mathrm{P}_{12}\right)=0.7$, Figs. $\mathrm{S} 1(\mathrm{~b})$ and $\left.\mathrm{S} 1(\mathrm{~d})\right)$. Since there is no additional source of information, a negative $\mathrm{P}_{66}$, i.e., a destructive addition in Eq. (3), is needed for an adequate theoretical representation of the BLS intensities.

The assignment of the observed branches and the appearance of the band gap (between modes $\mathbf{1}$ and $\mathbf{3}$ ) in Figure 3 can be ambiguous in the absence of rigorous theoretical calculations of the full BLS experiment. In fact, the experimental band gap concerns only branches with a high longitudinal character, whereas the theory predicts a smaller unidirectional stopband (Figs. 1 and 3). To provide more insight into the nature of the observed branches, maps of the displacement fields for the modes close to the first BZ boundary are displayed in Fig. 3(c) with increasing frequency at $q_{\Gamma \mathrm{M}}=0.0057 \mathrm{~nm}^{-1}$. Note that mode 2 close to the $\mathrm{M}$ point of the Brillouin zone is strongly longitudinal (vibration along the $\Gamma \mathrm{M}$ direction), whereas both modes $\mathbf{1}$ and 3 possess mixed polarization. Their motion corresponds to successive contraction/dilatation of both sides of the square, while the corners are kept almost fixed. In the evaluation of the BLS intensity (Eq. (3)), the main contribution to the strain arises from the second term for mode $\mathbf{1}$ and the first term for modes $\mathbf{2}$ and $\mathbf{3}$.

The strong diffraction and the occurrence of the BZ along $\Gamma \mathrm{X}$ at lower $q\left(=0.004 \mathrm{~nm}^{-1}\right)$ than for $\Gamma \mathrm{M}$ (Fig. 1) render the experimental access along $\Gamma \mathrm{X}$ ambiguous. To reduce the strong elastic scattering, we proceed with filled nanopores ${ }^{5,6}$ infiltrating the $\mathrm{hPnC}$ with Cargille (F) liquid (refractive index, $n_{\mathrm{F}}=1.513$ ) almost optically matching the ZPO matrix $\left(n_{Z}=1.51\right)$. Although the infiltration reduces the impedance contrast, the mismatch between corresponding sound velocities and densities of ZPO and $\mathbf{F}$ liquid is sufficient to allow an acoustic Bragg interference. Such band gap along two symmetry directions is displayed in the phonon dispersion of Figures 4(a) and 4(d).

To calculate the contribution of the liquid to the light scattering, we assume that the only strain in the liquid results from the dilatation, so the corresponding photoelastic parameters are taken to be $\mathrm{P}_{11}=\mathrm{P}_{12}$ (or $\mathrm{P}_{66}=0$ ) and called $\mathrm{P}$. Then, the expressions of the BLS intensities for phonon propagation along $\Gamma \mathrm{M}$ and $\Gamma \mathrm{X}$ become, respectively, 


$$
\begin{aligned}
\mathrm{I}_{\Gamma \mathrm{M}}= & \left|\mathrm{E}^{\mathrm{s}}\right|^{2} \propto \mid \frac{1}{\omega} \iint_{\text {solid part }} \mathrm{e}^{i \overrightarrow{\mathrm{q}} \overrightarrow{\mathrm{r}}} \times\left[\left(\mathrm{P}_{11}+\mathrm{P}_{12}\right) \times\left(\frac{\partial \mathrm{u}_{\mathrm{x}}}{\partial \mathrm{x}}+\frac{\partial \mathrm{u}_{\mathrm{y}}}{\partial \mathrm{y}}\right)-2 \times \mathrm{P}_{66} \times\left(\frac{\partial \mathrm{u}_{\mathrm{x}}}{\partial \mathrm{y}}+\frac{\partial \mathrm{u}_{\mathrm{y}}}{\partial \mathrm{x}}\right)\right] \\
& \times \mathrm{E}^{\mathrm{I} \mathrm{d} S}+\frac{1}{\omega} \iint_{\text {liquid part }} \mathrm{e}^{i \overrightarrow{\mathrm{q}} \overrightarrow{\mathrm{r}}} \times\left[2 \times \mathrm{P} \times\left(\frac{\partial \mathrm{u}_{\mathrm{x}}}{\partial \mathrm{x}}+\frac{\partial \mathrm{u}_{\mathrm{y}}}{\partial \mathrm{y}}\right)\right] \times\left.\mathrm{E}^{\mathrm{I}} \mathrm{dS}\right|^{2} \\
\mathrm{I}_{\Gamma \mathrm{X}}= & \left|\mathrm{E}^{\mathrm{s}}\right|^{2} \propto\left|\frac{1}{\omega} \iint_{\text {solid part }} \mathrm{e}^{i \overrightarrow{\mathrm{q}} \overrightarrow{\mathrm{r}}} \times\left[\left(\mathrm{P}_{12} \times \frac{\partial \mathrm{u}_{\mathrm{x}}}{\partial \mathrm{x}}+\mathrm{P}_{11} \times \frac{\partial \mathrm{u}_{\mathrm{y}}}{\partial \mathrm{y}}\right)\right] \times \mathrm{E}^{\mathrm{I}} \mathrm{d} \mathrm{S}+\frac{1}{\omega} \iint_{\text {liquid part }} \mathrm{e}^{i \overrightarrow{\mathrm{q}} \overrightarrow{\mathrm{r}}} \times\left[\mathrm{P} \times\left(\frac{\partial \mathrm{u}_{\mathrm{x}}}{\partial \mathrm{x}}+\frac{\partial \mathrm{u}_{\mathrm{y}}}{\partial \mathrm{y}}\right)\right] \times \mathrm{E}^{\mathrm{I}} \mathrm{dS}\right|^{2} .
\end{aligned}
$$

The theoretical dispersion curves for the filled-hPnC are considered in Figure 4 along $\Gamma \mathrm{X}$ and $\Gamma \mathrm{M}$ and are again unfolded into the second BZ; additionally, in Figure S2, we give the dispersion curves colored according to their longitudinal versus transverse character. In the calculations, the longitudinal sound velocity in $\mathbf{F}$ is fixed to its bulk value $\left(c_{\mathrm{f}}=1670 \mathrm{~m} / \mathrm{s}\right)$, while the acoustic velocities in the solid ZPO are fixed to $c_{L}=2900 \mathrm{~m} / \mathrm{s}$ and $c_{T}=1400 \mathrm{~m} / \mathrm{s}$ (Figs. S2(a)-S2(d)) or $c_{T}=1700 \mathrm{~m} / \mathrm{s}$ (Figs. $\mathrm{S} 2(\mathrm{e})-\mathrm{S} 2(\mathrm{~h})$ ), as in the case of the air-filled hPnC (Fig. S1). To investigate which longitudinal modes contribute significantly to the BLS spectra, the computation of their intensities includes now the photoelastic coefficient, $\mathrm{P}$, in $\mathbf{F}$ liquid as adjustable parameter (Eq. (4)). Good agreement with the experimental spectra was expectedly obtained with a higher $\mathrm{P}$ in the fluid compared to $\left(\mathrm{P}_{11}+\mathrm{P}_{12}\right)$ in the $\mathrm{ZPO}$ solid; $\mathrm{P} /\left(\mathrm{P}_{11}+\mathrm{P}_{12}\right)=4$ (Fig. S3). While both $c_{T}$ values lead to a good agreement between theory and experiment along $\Gamma \mathrm{X}$, the branches in the band diagram along $\Gamma \mathrm{M}$ are not represented equally well with either $c_{T}$ value; lowest (highest) branches favor the lower (higher) $c_{T}$ value (Figs. S2(b) and S2(d)). We note that the airfilled (Fig. 3) and infiltrated (Fig. 4) films used in this study are not the same and their fabrication can tolerate somewhat different $c_{T}$ values. Hence, a closer estimate of this quantity is not justified without additional physical arguments. Nevertheless, the full representation of the BLS experiment is the correct approach to yield the elastic and photoelastic parameters of the $\mathrm{hPnC}$ constituent components at nanoscale. ${ }^{28}$ We have also examined an alternative approach assuming a solidification of
F next to the wall. ${ }^{30} \mathrm{~A}$ thin $(\sim 10 \mathrm{~nm})$ solidified layer at the interface with low transverse acoustic velocity $(\sim 100 \mathrm{~m} / \mathrm{s})$ creates few additional features (flat branches) in the band diagram but are not identifiable among the experimental branches.

The displacement fields associated with the modes that contribute to BLS are presented in Figs. 4(c) and 4(f) for selected values of the wave vector. For instance, along $\Gamma X$, the mode at the frequency $1.02 \mathrm{GHz}$ and $q=0.0031 \mathrm{~nm}^{-1}$ is longitudinal and its contribution to BLS mainly arises from the fluid $\mathrm{hPnC}$ component. For $q=0.0057 \mathrm{~nm}^{-1}$, the modes at $1.42 \mathrm{GHz}$ and $1.63 \mathrm{GHz}$ are mainly longitudinal whereas the mode at $2.30 \mathrm{GHz}$ is of mixed character. We note that the peak termed $\mathbf{2}$ results from the contributions of two modes at $1.42 \mathrm{GHz}$ and $1.63 \mathrm{GHz}$. Similarly, in the case of $\Gamma \mathrm{M}$ direction, some of the peaks result from the contribution of two close modes (Fig. 4(e)). Overall, filling of the holes strongly impacts $\mathrm{hPnC}$ the band structure as well as the nature of the probed modes by BLS. It includes the change in $c_{\text {eff }}$ (see below), the penetration of the displacement field into the fluid, and the activation of high frequency modes along the $c_{\text {eff }}$-line.

The effective sound velocity, $c_{\text {eff }}$, at long wavelengths, obtained from the dispersion relations at sufficiently low $q$ 's (Figs. 4(a) and 4(d)), is distinct along the two symmetry directions. Indeed, the theory (Fig. 5) predicts about $13 \%$ higher value $\left(c_{\text {eff }}=2320 \mathrm{~m} / \mathrm{s}\right)$ along $\Gamma \mathrm{X}$ than along $\Gamma \mathrm{M}\left(c_{\text {eff }}=2040 \mathrm{~m} / \mathrm{s}\right)$. The effective sound velocity in the air-filled $\mathrm{hPnC}$ displays the same trend and the reduction compared to the filled counterpart is direction dependent, from about $\sim 15 \%$ along $\Gamma \mathrm{M}$ to
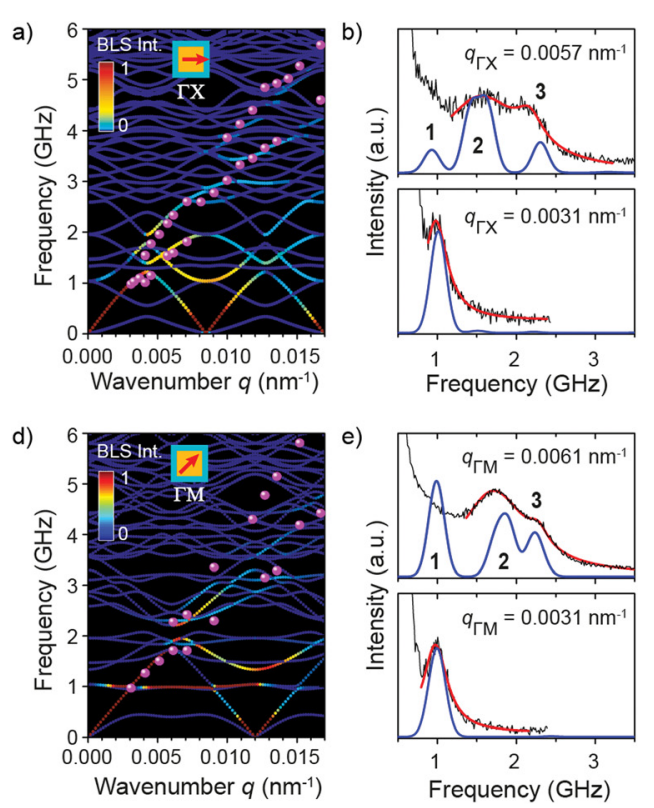
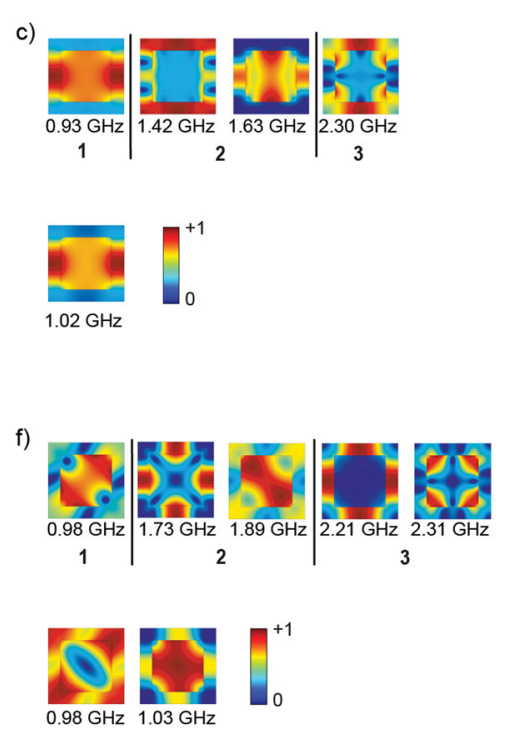

FIG. 4. The experimental (pink circles) and theoretical (lines) band diagrams (a) and (d), the experimental (black) and theoretical (blue) BLS spectra at two wave vectors, $q$ 's (b) and (e), and the displacement fields for the observed modes (c) and (f) of the filled hPnC along the $\Gamma \mathrm{X}(\mathrm{a})-(\mathrm{c})$ and $\Gamma \mathrm{M}$ (d)-(f) directions. The experimental spectra were represented by a sum of Lorentzian shapes (red lines in (b), (e)). The band diagrams are calculated for $c_{T}=1700 \mathrm{~m} / \mathrm{s}$. 


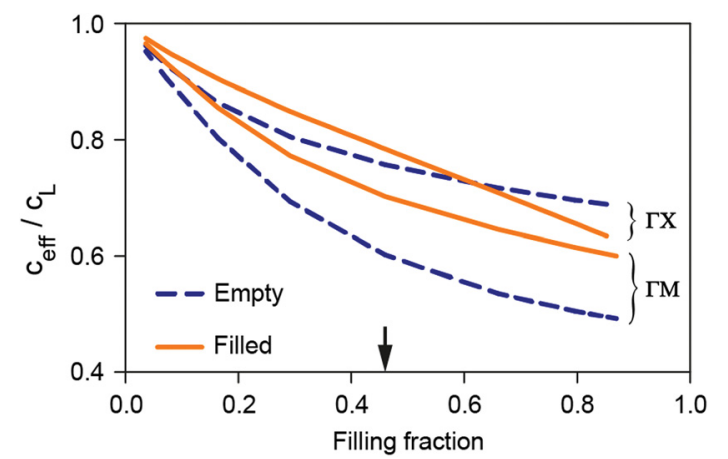

FIG. 5. Effective sound velocity ( $\left.c_{\text {eff }}\right)$ normalized to the longitudinal sound velocity of the matrix $\left(c_{\mathrm{L}}\right)$ along $\Gamma \mathrm{X}$ and $\Gamma \mathrm{M}$. The arrow indicates the filling fraction of the present $\mathrm{hPnC}$.

$\sim 5 \%$ along $\Gamma X$ (Fig. 5). The observed anisotropy of the effective sound velocity is expected in view of the symmetry of the square lattice, in contrast to an isotropic behavior in a triangular lattice $\mathrm{hPnC}$ with circular nanopores. Triggered by this unpredicted observation, we computed $c_{\text {eff }}$ as a function of the filling fraction also for two filling liquids with different fluid velocities, $c_{\mathrm{f}}(1670 \mathrm{~m} / \mathrm{s}$ and $1000 \mathrm{~m} / \mathrm{s}$ in Fig. S4). There is no systematic variation of $c_{\text {eff }}$ with $c_{\mathrm{f}}$, and certainly the common Wood's law fails.

Summing up, the present experimental and theoretical study demonstrates the effect of the shape of the holes on the direction-dependent hypersonic phononic properties of a cavity with square lattice symmetry fabricated by femtosecond laser pulse-induced two photon polymerization technique. Filling the holes with a fluid strongly modifies the phononic band diagram being a necessary condition for sensor applications. ${ }^{39}$ Yet, we have theoretically examined the tuning sensitivity to the filling material, fraction, and shape of the inclusions in square lattice phononics that calls for experimental verification. The full representation of the experiment (band structure and intensities) is necessary to yield a unique identification of all probed vibration modes, the direction dependent effective medium sound velocity, and the longitudinal and transverse moduli of the constituent material at nanoscale. A detailed understanding of phonon propagation in periodic structures is a precondition to access fundamental concepts of strong phonon-photon interactions and thermal properties. ${ }^{40,41}$

The support of Kazakhstan Ministry of Education and Science (Nos. 0662 \& 5385/GF4), Nazarbayev University and Government of the Russian Federation (No. 14.B25.31.0019) is acknowledged. E.A. acknowledges the support by ERC SuPro 340391. D. Perevoznik was supported by FP7-MC and ITN Nano2Fun (GA 607721). G.F. also acknowledges the partial support by Aristeia Program (EU, GSST Greece).

${ }^{1}$ R. H. Olsson et al., in International Ultrasonics Symposium, Rome, Italy, 20-23 September 2009 (IEEE, 2009), p. 1150.

${ }^{2}$ S. Mohammadi, A. A. Eftekhar, A. Khelif, W. D. Hunt, and A. Adibi, Appl. Phys. Lett. 92, 221905 (2008).

${ }^{3}$ J.-H. Lee, C. Y. Koh, J. P. Singer, S. J. Jeon, M. Maldovan, O. Stein, and E. L. Thomas, Adv. Mater. 26, 532 (2014).

${ }^{4}$ M. Ziaei-Moayyed, M. F. Su, C. Reinke, I. F. El-Kady, and R. H. Olsson, in 24th International Conference on Micro Electro Mechanical Systems, Cancun, Mexico, 23-27 January (IEEE, 2011), p. 1377.
${ }^{5}$ W. Cheng, J. Wang, U. Jonas, G. Fytas, and N. Stefanou, Nat. Mater. 5, 830 (2006).

${ }^{6}$ T. Gorishnyy, J. Jang, C. Y. Koh, and E. L. Thomas, Appl. Phys. Lett. 91, 121915 (2007).

${ }^{7}$ T. Still, W. Cheng, M. Retsch, R. Sainidou, J. Wang, U. Jonas, N. Stefanou, and G. Fytas, Phys. Rev. Lett. 100, 194301 (2008).

${ }^{8}$ A. V. Akimov, Y. Tnaka, A. B. Pevtsov, S. F. Kaplan, V. G. Golubev, S. Tamura, D. R. Yakovlev, and M. Bayer, Phys. Rev. Lett. 101, 033902 (2008). ${ }^{9}$ P. M. Walker, J. S. Sharp, A. V. Akimov, and A. J. Kent, Appl. Phys. Lett. 97, 073106 (2010).

${ }^{10}$ V. L. Zhang, F. S. Ma, H. H. Pan, C. S. Lin, H. S. Lim, S. C. Ng, M. H. Kouk, S. Jain, and A. O. Adeyeye, Appl. Phys. Lett. 100, 163118 (2012).

${ }^{11}$ G. Zhu, N. Z. Swinteck, S. Wu, J. S. Zhang, H. Pan, J. D. Bass, P. A. Deymier, D. Banerjee, and K. Yano, Phys. Rev. B 88, 144307 (2013).

${ }^{12}$ D. Schneider, F. Liaqat, E. H. El Boudouti, O. El Abouti, W. Tremel, H.-J. Butt, B. Djafari-Rouhani, and G. Fytas, Phys. Rev. Lett. 111, 164301 (2013).

${ }^{13}$ S. Wu, G. Zhu, J. S. Zhang, D. Banerjee, J. D. Bass, C. Ling, and K. Yano, Phys. Chem. Chem. Phys. 16, 8921 (2014).

${ }^{14}$ P. Beltramo, D. Schneider, G. Fytas, and E. M. Furst, Phys. Rev. Lett. 113, 205503 (2014).

${ }^{15}$ D. F. Goettler, M. F. Su, C. M. Reinke, S. Alaie, P. E. Hopkins, R. H. Olsson III, I. El-Kady, and Z. C. Leseman, AIP Adv. 1, 042001 (2011).

${ }^{16}$ V. L. Zhang, C. G. Hou, H. H. Pan, F. S. Ma, M. H. Kuok, H. S. Lim, S. C. Ng, M. G. Cottam, M. Jamali, and H. Yang, Appl. Phys. Lett. 101, 053102 (2012).

${ }^{17} \mathrm{~J}$. Li and S. Yang, Microelectron. Eng. 128, 7 (2014).

${ }^{18}$ T. Gorishnyy, C. K. Ullal, M. Maldovan, G. Fytas, and E. L. Thomas, Phys. Rev. Lett. 94, 115501 (2005).

${ }^{19}$ S. Maruo, O. Nakamura, and S. Kawata, Opt. Lett. 22, 132 (1997).

${ }^{20}$ J. Serbin, A. Egbert, A. Ostendorf, B. Chichkov, R. Houbertz, G. Domann, J. Schulz, C. Cronauer, L. Fröhlich, and M. Popall, Opt. Lett. 28, 301 (2003).

${ }^{21}$ L. Montemayor, V. Chernow, and J. R. Greer, MRS Bull. 40, 1122 (2015).

${ }^{22}$ H. B. Sun, S. Matsuo, and H. Misawa, Appl. Phys. Lett. 74, 786 (1999).

${ }^{23}$ J. Serbin, A. Ovsianikov, and B. N. Chichkov, Opt. Express 12, 5221 (2004).

${ }^{24}$ M. Deubel, G. von Freymann, M. Wegener, S. Pereira, and K. Busch, Nat. Mater. 3, 444 (2004).

${ }^{25}$ R. Guo, Zh. Li, Z. Jiang, D. Yuan, W. Huang, and A. Xia, J. Opt. A: Pure Appl. Opt. 7, 396 (2005).

${ }^{26}$ A. Ovsianikov, J. Viertl, B. Chichkov, M. Oubaha, B. MacCraith, I. Sakellari, A. Giakoumaki, D. Gray, M. Vamvakaki, M. Farsari, and C. Fotakis, ACS Nano 2, 2257 (2008).

${ }^{27}$ M. V. Rybin, I. I. Shishkin, K. B. Samusev, P. A. Belov, Y. S. Kivshar, R. V. Kiyan, B. Chichkov, and M. F. Limonov, Crystals 5, 61 (2015).

${ }^{28}$ F. Kargar, S. Ramirez, B. Debnath, H. Malekpour, R. K. Lake, and A. A. Balandin, Appl. Phys. Lett. 107, 171904 (2015).

${ }^{29}$ B. Graczykowski, M. Sledzinska, F. Alzina, J. Gomis-Bresco, J. S. Reparaz, M. R. Wagner, and C. M. Sotomayor Torres, Phys. Rev. B 91, 075414 (2015).

${ }^{30}$ A. Sato, Y. Pennec, T. Yanagishita, H. Masuda, W. Knollm, B. DjafariRouhani, and G. Fytas, New J. Phys. 14, 113032 (2012).

${ }^{31}$ J. Vasseur, B. Djafari-Rouhani, L. Dobrzynski, M. S. Kushwaha, and P. Halevi, J. Phys.: Condens. Matter 6, 8759 (1994).

${ }^{32}$ C. Goffaux and J. P. Vigneron, Phys. Rev. B 64, 075118 (2001).

${ }^{33}$ S. Babaee, N. Viard, P. Wang, X. Fang, and K. Bertoldi, Adv. Mater. 28, 1631 (2016).

${ }^{34}$ M. Farsari and B. N. Chichkov, Nat. Photonics 3, 450 (2009).

${ }^{35}$ P. Voudouris, J. Choi, N. Gomopoulos, R. Sainidou, H. Dong, K. Matyjaszewski, M. R. Bockstaller, and G. Fytas, ACS Nano 5, 5746 (2011).

${ }^{36}$ See supplementary material at http://dx.doi.org/10.1063/1.4949013 for details of phonon dispersions behaviors from the photo-elastic mechanism of Brillouin light scattering as a function of the elastic and photoelastic parameters.

${ }^{37}$ D. Schneider, F. Liaqat, E. H. El Boudouti, Y. El Hassouani, B. DjafariRouhani, W. Tremel, H.-J. Butt, and G. Fytas, Nano Lett. 12, 3101 (2012).

${ }^{38}$ O. L. Lazarenkova and A. A. Balandin, Superlattices Microstruct. 33, 95 (2003).

${ }^{39}$ S. Amoudache, Y. Pennec, B. Djafari-Rouhani, A. Khater, R. Lucklum, and R. Tigrine, J. Appl. Phys. 115, 134503 (2014).

${ }^{40}$ M. M. Maldovan, Nature 503, 209 (2013).

${ }^{41}$ S. Volz, J. Ordonez-Miranda, A. Shchepetov, M. Prunnila, J. Ahopelto, T. Pezeril, G. Vaudel, V. Gusev, P. Ruello, E. M. Weig, M. Schubert, M. Hettich, M. Grossman, T. Dekorsy, F. Alzina, B. Graczykowski, E. Chavez-Angel, J. S. Reparaz, M. R. Wagner, C. M. Sotomayor-Torres, S. Xiong, S. Neogi, and D. Donadio, Eur. Phys. J. B 89, 15 (2016). 